\title{
Ischemic colitis and Atrial septal aneurysm as a potential source for systemic thromboembolism
}

\author{
Eihab Subahi ${ }^{1}$, Narinder Kumar ${ }^{1}$, Abdulwahab Hamid ${ }^{1}$, Mohamed Elmahadi ${ }^{1}$, and \\ mohamed yassin ${ }^{2}$ \\ ${ }^{1}$ Hamad Medical Corporation \\ ${ }^{2} \mathrm{HMC}$ NCCCR
}

September 11, 2020

\begin{abstract}
The atrial septal aneurysm is a rare cardiac abnormality that is usually detected during routine echocardiography or evaluation of cases with thromboembolism. We report a 62 years old male who presented with abdominal pain and bleeding per rectum found to have ischemic colitis and atrial septal aneurysm on transesophageal echocardiogram.
\end{abstract}

Ischemic colitis and Atrial septal aneurysm as a potential source for systemic thromboembolism Eihab A. Subahi ${ }^{1}$, Narinder Kumar ${ }^{2}$, Abdulwahab M. Hamid ${ }^{3}$, Mohamed S. Elmahadi ${ }^{4}$, Mohamed A. Yassin ${ }^{5}$

1. Department of Internal Medicine, Hamad Medical Corporation, Doha, Qatar

2. Department of cardiology, Alkhor Hospital, Hamad Medical Corporation, Doha, Qatar

3. Department of Gastroenterology and Hepatology, Alkhor Hospital, Hamad Medical Corporation, Doha, Qatar

4. Department of General Medicine, Alkhor Hospital, Hamad Medical Corporation, Doha, Qatar

5. Department of Medical Oncology, National center for Cancer Care and Research Corresponding authors: Dr. Eihab A. Subahi

Department of Internal Medicine,

Hamad Medical Corporation,

Doha, Qatar

P.O 3050

Mobile: +97433189367

ehabsubahi@yahoo.com

ehabsubahi7@gamil.com

Short title: Ischemic colitis and atrial septal aneurysm

Keywords: Aneurysm. Colitis. thromboembolism

Key clinical massage

The atrial septal aneurysm is a rare cardiac abnormality with thromboembolic potential and should be considered in a patient with ischemic colitis with no obvious risk factors. 


\section{Abstract}

The atrial septal aneurysm is a rare cardiac abnormality that is usually detected during routine echocardiography or evaluation of cases with thromboembolism. It may be isolated or associated with other cardiac defects i.e. most often patent foramen ovale. Atrial arrhythmias and arterial embolisms are associated with complications that must be treated with anticoagulants. We report a 62 years old male who presented with abdominal pain and bleeding per rectum found to have ischemic colitis and atrial septal aneurysm on transesophageal echocardiogram, systemic thromboembolism is one of the complications of atrial septal aneurysm most of the reported cases are associated with stroke, TIA and renal emboli. our patient treated with anticoagulation, Rivaroxaban.

\section{Introduction}

An atrial septal aneurysm is defined as "redundant and mobile interatrial septal tissue in the region of the fossa ovalis with a phasic excursion of at least 10 to $15 \mathrm{~mm}$ during the cardiorespiratory cycle "(1).it can be classified according to their intrusion into the left or right atrium and according to their motion during the respiratory cycle (1).the aneurysm may either protrude preeminently into the right or left atrium or produce striking oscillation into the atrial cavities during the respiration according to variation in the pressure differences between the atria (1)(2). atrial septal aneurysm is most commonly discovered incidentally during a routine evaluation, but in some populations, it can be associated with systemic thromboembolism (1)(3)(4) and intracardiac shunt if it is associated with one or more atrial septal defects, the commonest association is patent foramen ovale (5).

Diagnosis of atrial septal aneurysm usually through a transthoracic echocardiogram, a however transesophageal echocardiogram is more sensitive as the interatrial septum can be visualized more consistently (6).

\section{Case presentation}

we report 62 years old male who presented to our facility with one-day history of severe lower abdominal pain associated with fresh bleeding per rectum. the patient had a history of hypertension and diabetes for a long time controlled with medications. upon presentation, the patient was in pain but conscious, alert, oriented, afebrile, and vitally stable. The abdominal exam was soft, lax with mild lower abdominal tenderness, the digital rectal examination was positive for blood. The cardiac exam was normal. his blood works showed mild neutrophilic leukocytosis and normal hemoglobin level. other labs with normal limits. Autoimmune and thrombophilia workup were both negative, viral serology for hepatitis B, C and HIV were negative.

CT scan of abdomen and pelvis with contrast showed diffused colitis involving entire descending colon with peri-colonic fat stranding and possible reduced bowel wall perfusion with poor contrast filling of distal branches of the left colic artery but without major vascular occlusions or stenosis which goes with the possibility of ischemic colitis (pictures 1 and 2). Colonoscopy was done and showed blushed mucosa goes with ischemia. biopsy result confirmed the diagnosis of ischemic colitis which showed necrotic bowel mucosa with fibrin thrombi and fibrinous material (pictures 3 and 4). Patient underwent 12 leads Electrocardiogram which was sinus and no arrhythmias, transthoracic Echocardiogram showed mild mitral valve regurgitation, mild to moderate tricuspid valve regurgitation, and suspicion of an atrial septal aneurysm with no obvious shunt across it. Bubble contrast Echocardiogram was negative for shunt across the atrial septum and the left atrium was clear with no thrombus.

Transesophageal echocardiogram showed atrial septal aneurysm, but was no patent foramen ovale or any other atrial septal defects (pictures 5 and 6). bilateral lower limb doppler both were negative for deep veins thrombosis. 48 hours Holter was negative for atrial fibrillations. The patient was counseled about the conventional anticoagulation Vs DOAC (direct oral anticoagulation), pros and cons were explained to him and he chose to be in DOAC. he was started on Rivaroxaban $15 \mathrm{mg}$ twice per day for 21 days then 20 mg daily and discharge on the same regimen. he was followed after 1-week form the date of discharge and there were no complications and he was compliant with his medication. till writing this case there was no 
documented complications or emergency visit for any reason.

Discussion

Our patient suffered from ischemic colitis which was proven by colonoscopy and tissue biopsy. His CT scan finding goes more with the embolic nature of disease rather than diffused atherosclerosis, and the only risk factor was atrial septal aneurysm which is a potential risk factor for cardiogenic embolism. After a discussion with cardiology and gastroenterology, our patient was labeled as arterial embolization with ischemic colitis secondary to an atrial septal aneurysm and was started on anticoagulation with Rivaroxaban (off label).

Atrial septal aneurysm manifestations can be either atrial arrhythmias or arterial embolisms. It can act as a source of arrhythmic focus leading to atrial tachycardias (6)(7). Arterial embolism is a well-known complication, different studies had shown a significant association between the atrial septal aneurysm and arterial embolism(1)(3)(4).

The echocardiogram is a gold stander diagnostic tool for an atrial septal aneurysm which is usually discovered during routine tests or during the evaluation of cardioembolic stroke or arterial embolisms. comparing transthoracic echocardiogram with transesophageal, the last one showed to be more sensitive in picking up atrial septal aneurysm(1)(6). other modalities like cardiac tomography and cardiac magmatic resonance showed to be useful as well for the diagnosis(8)(9).

Treatment lines for this pathology vary according to the presentation and associated structural cardiac anomalies, in an asymptomatic patient with isolated atrial septal aneurysm no specific treatment is required after ruling out intracardiac thrombus. In other hands, in a patient with cryptogenic stroke and presence of isolated atrial septal aneurysm options including medical therapy with antiplatelets or anticoagulation as in case of recurrent stroke while taking antiplatelets. rarely surgical excision of the defect is considered in a patient with a recurrent stroke whom antiplatelet or warfarin fail to prevent stroke recurrence or in patients with a large left to right shunt leading to right heart enlargement. Percutaneous device closure is also rarely performed as well(10).

Furthermore, in patients with atrial arrhythmias and embolic episodes, the preferable treatment is oral anticoagulation for secondary prevention. Most the authorities recommend traditional anticoagulation like in the case of renal artery emboli limited to one of the renal arteries or segmental branches (11). Embolectomy is another option in case of bilateral renal artery embolism if the patient suitable for the procedure. Lastly, intra-abdominal thrombolysis is also a valid option in such cases(12). In the patient with stroke or TIA American heart association/ American stroke association has recommended to use aspirin for secondary prevention and warfarin can be used in high-risk patients(13). And in other case reports of stroke associate with atrial septal aneurysm anticoagulation was employed as well(3)(14).

Moreover, in one case report a patient with paroxysmal atrial fibrillation developed intracardiac thrombus after found to had an atrial septal aneurysm and followed for 3 years. The patient started on warfarin, six months later no thrombus was found in repeated echocardiogram but the defect persists despite adequate anticoagulation later patient offer surgery (15). Till now there is no reported case in literature of ischemic colitis associated with an atrial septal aneurysm and the uses of DOAC on such cases and on systemic arterial embolization secondary to atrial septal aneurysm has not been studied as well.

\section{Conclusion}

It is concluded that in a patient with ischemic colitis and no obvious risk factors atrial septal aneurysm is a cardiac abnormality with thromboembolic potential and should be considered.

Acknowledgment

The authors would like to acknowledge the internal medicine residency program for their support.

Statement of Ethics

The patient consented to the publication of his case. 
Disclosure statement

The authors have no conflict of interest.

Authors contributions

Dr. Eihab A. Subahi and Dr. Mohamed A. Yassin wrote and edited the manuscript.

Dr. Narinder Kumar performed the Echocardiogram and provided us with labeled pictures of the cardiac lesion. Dr Abdulwahab M. Hamid perform the colonoscopy and provided us with labeled pictures of the colonoscopy. Dr. Mohamed S. Elmahadi was in charge of the clinical care.

\section{References}

1. Pearson AC, Nagelhout D, Castello R, Gomez CR, Labovitz AJ. Atrial septal aneurysm and stroke: A transesophageal echocardiographic study. J Am Coll Cardiol [Internet]. 1991 Nov 1 [cited 2020 Aug 22];18(5):1223-9. Available from: https://pubmed.ncbi.nlm.nih.gov/1918699/

2. Belkin RN, Kisslo J. Atrial septal aneurysm: Recognition and clinical relevance. Am Heart J [Internet]. 1990 [cited 2020 Aug 22];120(4):948-57. Available from: https://pubmed.ncbi.nlm.nih.gov/2220548/

3. Gallet B, Malergue MC, Adams C, Saudemont JP, Collot AM, Druon MC, et al. Atrial septal aneurysm - a potential cause of systemic embolism. An echocardiographic study. Br Heart J [Internet]. 1985 [cited 2020 Aug 22];53(3):292-7. Available from:/pmc/articles/PMC481758/?report=abstract

4. Belkin RN, Hurwitz BJ, Kisslo J. Atrial septal aneurysm: association with cerebrovascular and peripheral embolic events. Stroke [Internet]. 1987 Sep [cited 2020 Aug 22];18(5):856-62. Available from: https://www.ahajournals.org/doi/10.1161/01.STR.18.5.856

5. Silver MD, Dorsey JS. Aneurysms of the septum primum in adults. Arch Pathol Lab Med. 1978;102:62-65. [Internet]. [cited 2020 Aug 22]. Available from: http://www.sciepub.com/reference/152236

6. Mügge A, Daniel WG, Angermann C, Spes C, Khandheria BK, Kronzon I, et al. Atrial septal aneurysm in adult patients: A multicenter study using transthoracic and transesophageal echocardiography. Circulation [Internet]. 1995 Jun 1 [cited 2020 Aug 22];91(11):2785-92. Available from: https://pubmed.ncbi.nlm.nih.gov/7758185/

7. Hanley PC, Tajik AJ, Hynes JK, Edwards WD, Reeder GS, Hagler DJ, et al. Diagnosis and classification of atrial septal aneurysm by two-dimensional echocardiography: Report of 80 consecutive cases. J Am Coll Cardiol. 1985 Dec 1;6(6):1370-82.

8. Hur J, Kim YJ, Lee HJ, Ha JW, Heo JH, Choi EY, et al. Cardiac computed tomographic angiography for detection of cardiac sources of embolism in stroke patients. Stroke [Internet]. 2009 Jun 1 [cited 2020 Aug 22];40(6):2073-8. Available from: https://yonsei.pure.elsevier.com/en/publications/cardiac-computedtomographic-angiography-for-detection-of-cardiac

9. Saremi F, Channual S, Raney A, Gurudevan S V., Narula J, Fowler S, et al. Imaging of patent foramen ovale with 64-section multidetector CT. Radiology [Internet]. 2008 Nov [cited 2020 Aug 22];249(2):483-92. Available from: https://pubmed.ncbi.nlm.nih.gov/18780828/

10. Burger AJ, Sherman HB, Charlamb MJ. Low incidence of embolic strokes with atrial septal aneurysms: A prospective, long-term study. Am Heart J [Internet]. 2000 Jan 1 [cited 2020 Aug 22];139(1):149-52. Available from: https://linkinghub.elsevier.com/retrieve/pii/S0002870300700226

11. Renal artery embolism: clinical features and long-term follow-up of 17 cases - PubMed [Internet]. [cited 2020 Aug 22]. Available from: https://pubmed.ncbi.nlm.nih.gov/697226/

12. Braun DR, Sawczuk IS, Axelrod SA. Idiopathic renal infarction. Urology [Internet]. 1995 [cited 2020 Aug 22];45(1):142-5. Available from: https://pubmed.ncbi.nlm.nih.gov/7817469/ 
13. Sacco RL, Adams R, Albers G, Alberts MJ, Benavente O, Furie K, et al. Guidelines for prevention of stroke in patients with ischemic stroke or transient ischemic attack: A statement for healthcare professionals from the American Heart Association/American Stroke Association council on stroke - Co-sponsored by the council on cardiovascular radiology and intervention. The American Academy of Neurology affirms the value of this guideline [Internet]. Vol. 37, Stroke. Lippincott Williams and Wilkins; 2006 [cited 2020 Aug 22]. p. 577-617. Available from: https://pubmed.ncbi.nlm.nih.gov/16432246/

14. Giuseppe Di Pasquale, Alvaro Andreoli PG, Paola Dominici and GP. Cardioembolic Stroke From Atrial Septal Aneurysm. AHA journals. 1988;19(5):640-3.

15. Shinohara T, Kimura T, Yoshizu H, Ohsuzu F. Three-Year Follow-up of an Atrial Septal Aneurysm. Ann Thorac Surg [Internet]. 2001 May 1 [cited 2020 Aug 22];71(5):1672-3. Available from: http://www.annalsthoracicsurgery.org/article/S0003497500023055/fulltext

List of figures

Picture number Description

Picture 1 Diffuse circumferential wall thickening of the large colon involving distal third of transverse colon (Blue a

Picture 2 Patent main left colic artery, with evidence of poor contrast filling of its distal branches (Blue arrow) wit

Picture $3 \quad$ yellow arrow showed bluish colonic mucosa suggesting ischemic colitis

Picture $4 \quad$ yellow arrow pointed to bluish colonic mucosa suggesting ischemic colitis

Picture $5 \quad$ Transesophageal echocardiogram and Yellow arrow showed atrial septal aneurysm (ASA).

Picture $6 \quad$ Transesophageal echocardiogram and Yellow arrow showed atrial septal aneurysm (ASA). 


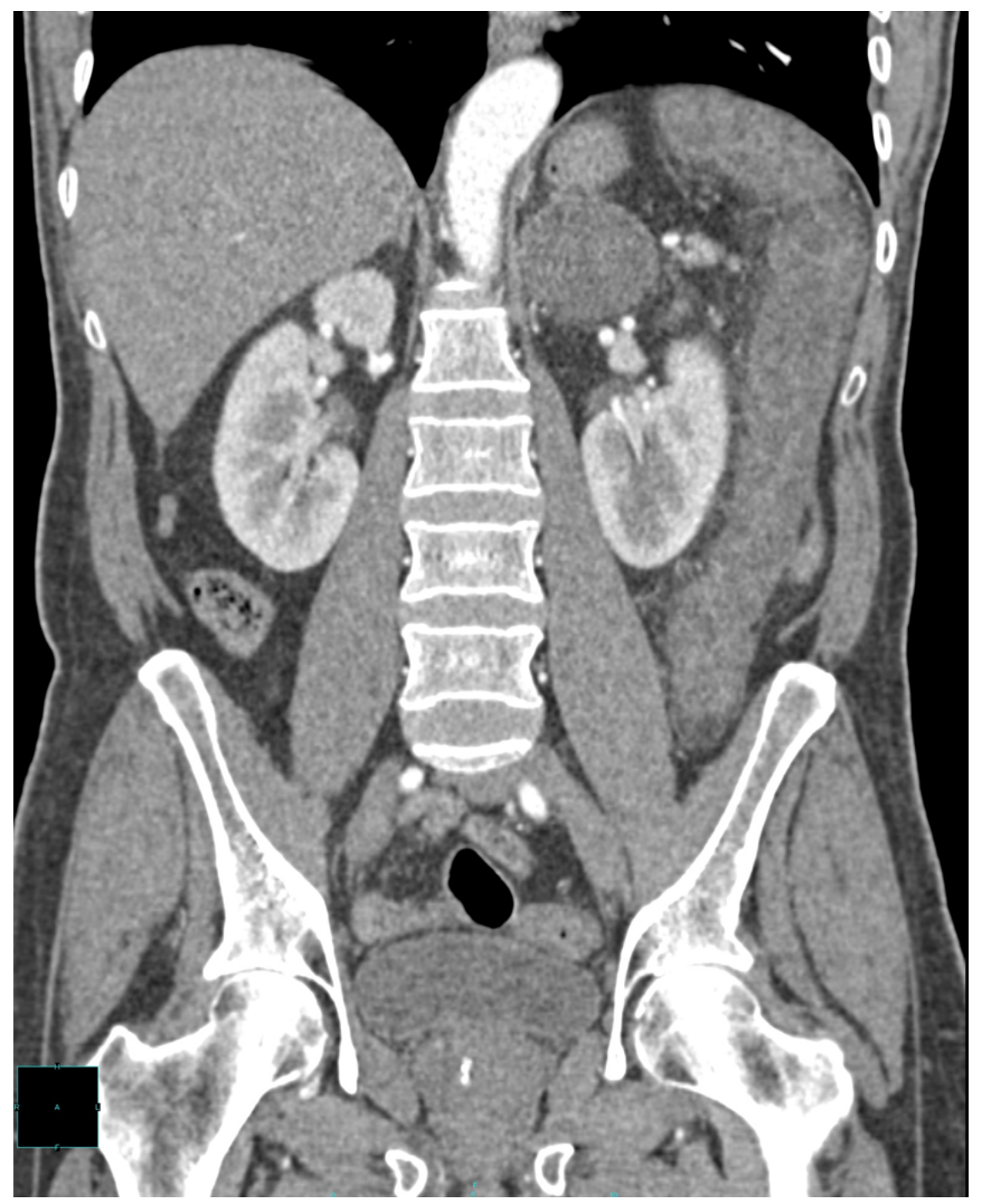

Picture 1: Diffuse circumferential wall thickening of the large colon involving distal third of transverse colon (Blue arrow), splenic flexure (Green arrow) and the entire descending colon (white arrow), associated with reduce mural enhancement and surrounding peri-colonic fat standing 


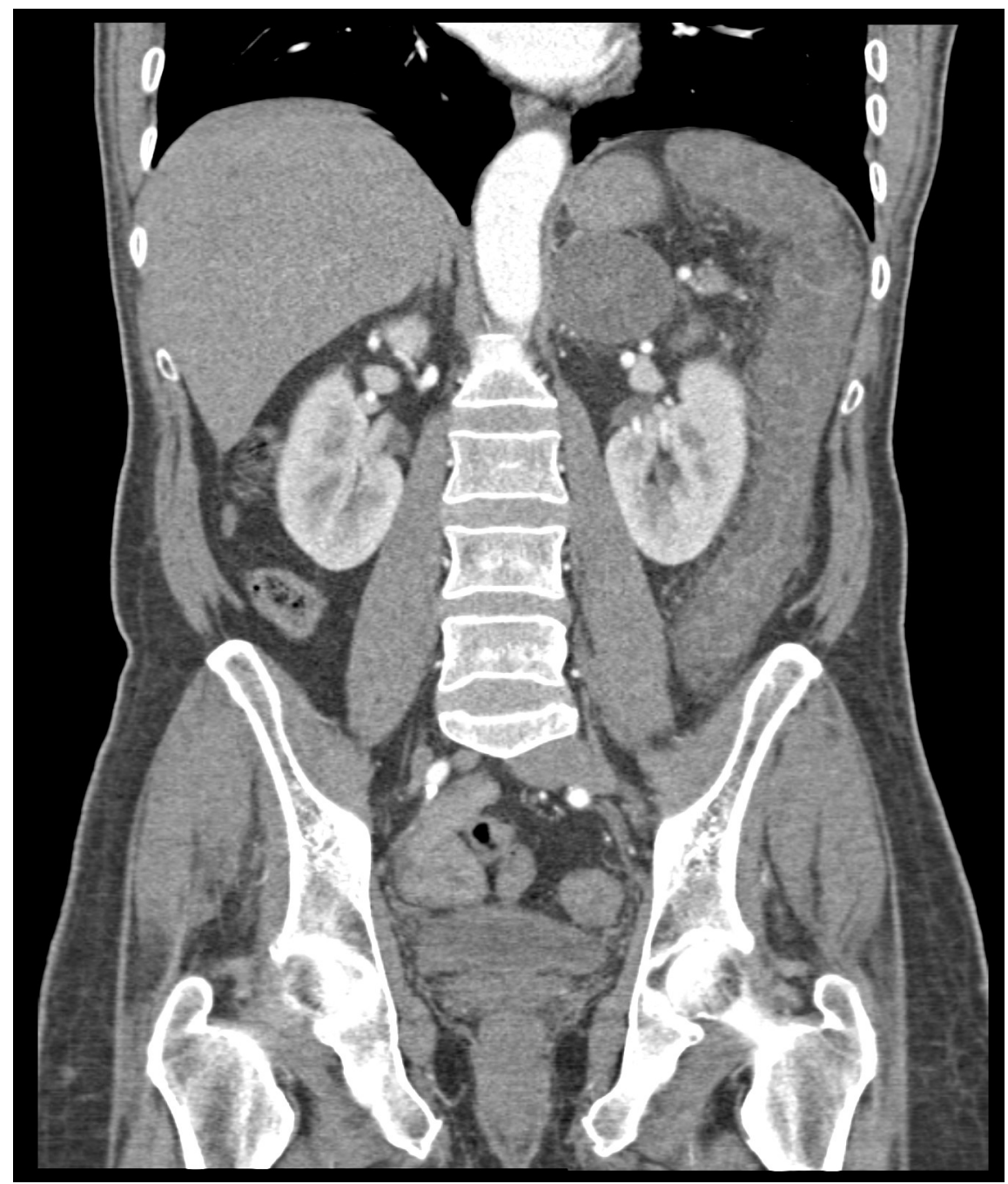

Picture 2: Patent main left colic artery, with evidence of poor contrast filling of its distal branches (Blue arrow) with poorly perfused vas recta and associated overall poor bowel wall enhancement. 


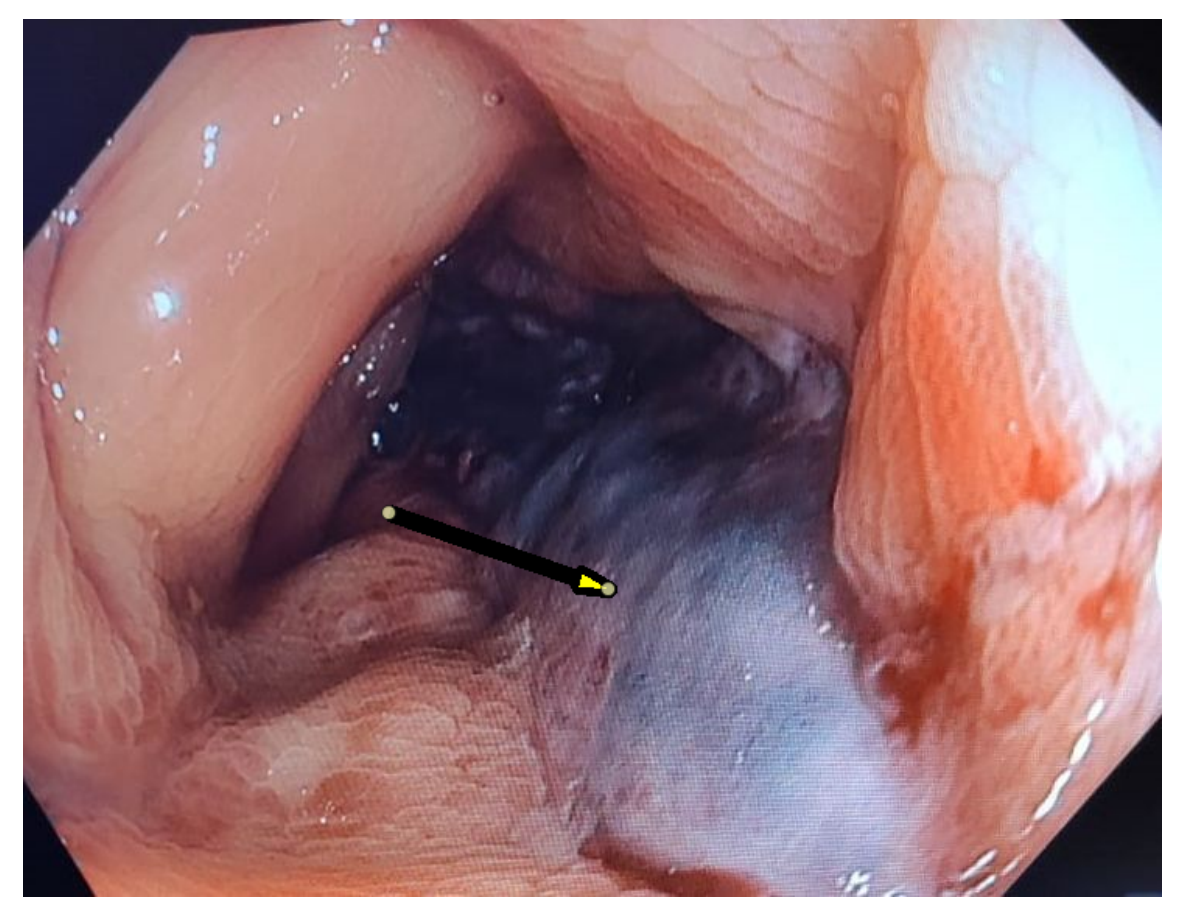

Picture 3: yellow arrow showed bluish colonic mucosa suggesting ischemic colitis

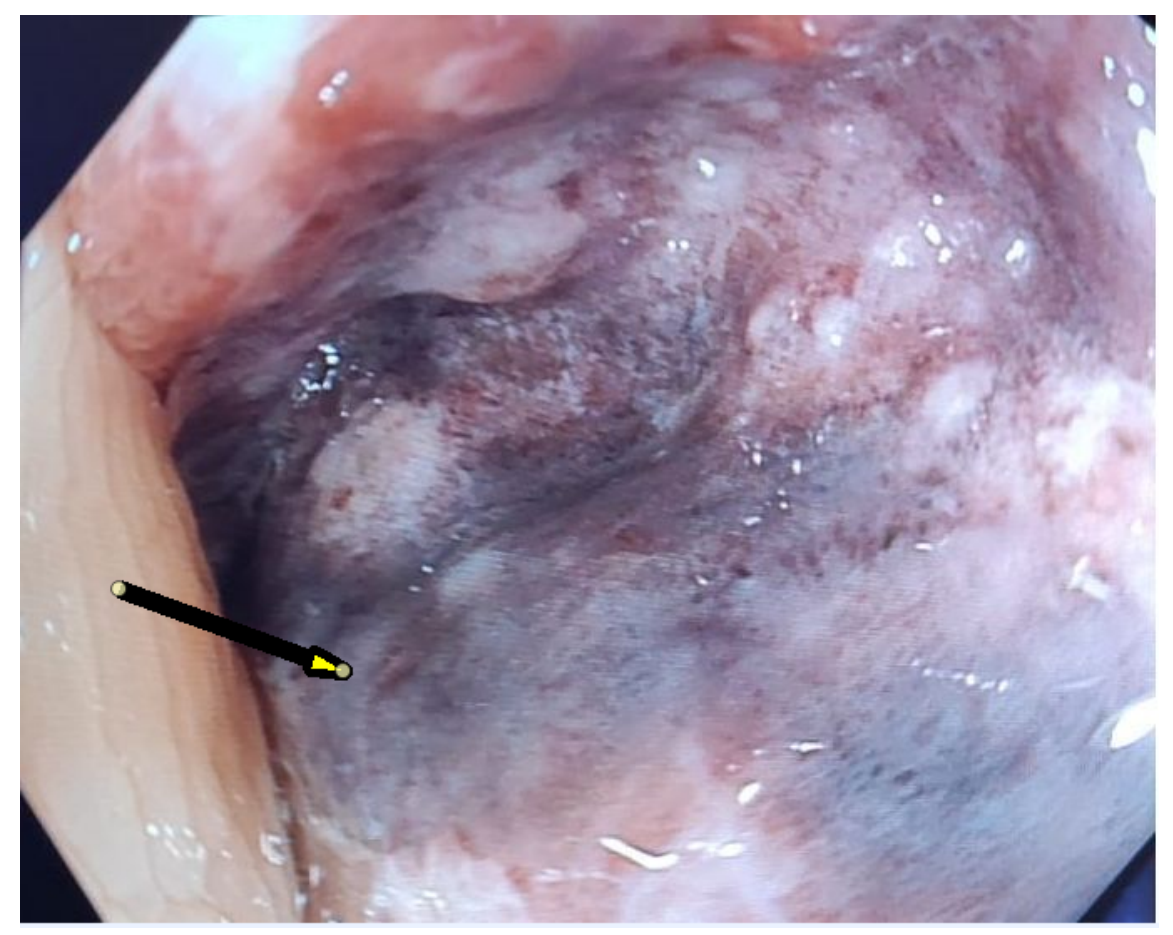

Picture 4: yellow arrow pointed to bluish colonic mucosa suggesting ischemic colitis 


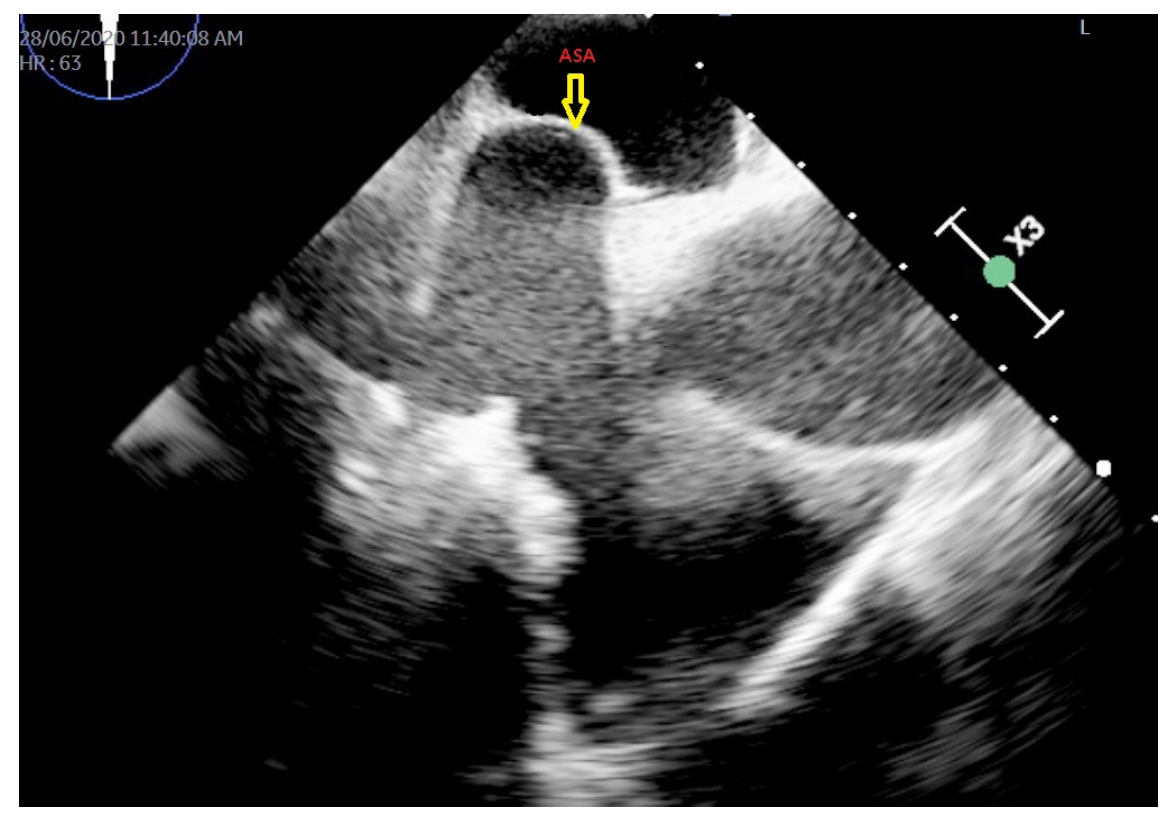

Picture 5: Transesophageal echocardiogram and Yellow arrow showed atrial septal aneurysm (ASA)

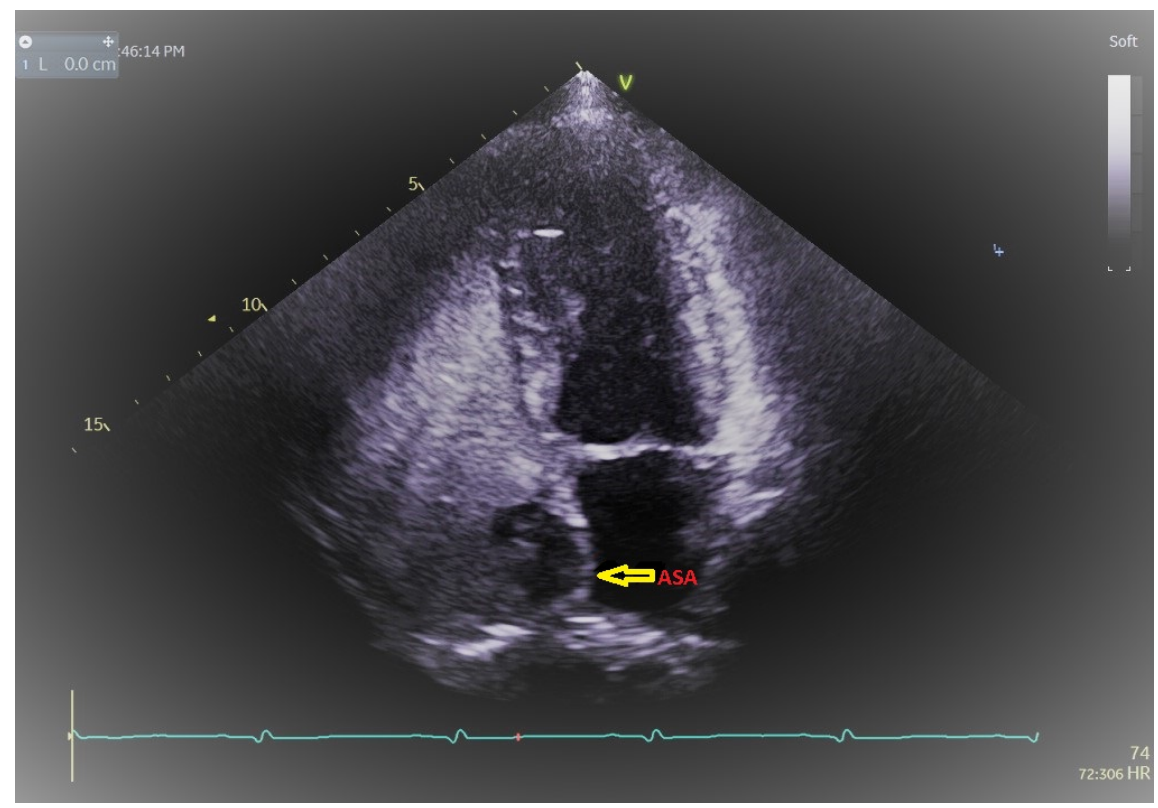

Picture 6: Transesophageal echocardiogram and Yellow arrow showed atrial septal aneurysm (ASA) Picture 3: yellow arrow showed bluish colonic mucosa suggesting ischemic colitis 